\title{
Mixed Road Surface Model for Driving Assistance Systems
}

\author{
Florin Oniga, Radu Danescu, and Sergiu Nedevschi \\ Computer Science Department \\ Technical University of Cluj-Napoca, Romania \\ \{florin.oniga, radu.danescu, sergiu.nedevschi \}@ cs.utcluj.ro
}

\begin{abstract}
The problem of road/obstacle separation of 3D points is revisited in this paper, in the context of driving assistance systems and dense stereovision. We propose some measures to cope with scenarios where only few road points are recovered in 3D, or the road has an atypical geometry. When a non-planar road model is employed, e.g. a quadratic road surface, the vertical profile of the road is usually reliable for the $3 D$ region that contains the road inliers. Therefore, using this surface for obstacle/road separation outside the inliers region is less reliable. Our solution is a mixed road model: the quadratic road surface is filtered temporally and extended with a planar patch to cover the whole space of interest. The planar surface used for extension is computed from the parameters of the quadratic surface.
\end{abstract}

Keywords- road surface estimation, obstacle/road separation, dense stereovision

\section{INTRODUCTION}

Obstacle detection through image processing has followed two main trends: single-camera based detection and two (or more) camera based detection (stereovision-based detection). The stereovision-based approaches have the advantage of directly measuring the 3D coordinates of an image feature, this feature being anything from a point to a complex structure. The main constraints concerning stereovision applications are to minimize the calibration and stereo-matching errors in order to increase the measurements accuracy and to reduce the complexity of stereo-correlation process.

An important part in the obstacle detection process is the separation of the obstacle points from the road points. Some of the obstacle detection methods are based on the flat road assumption [1,2]. This is a poor model since deviations from the flat road model may be as large as or larger than the obstacles we wish to detect. In consequence the separation and the $3 \mathrm{D}$ objects position estimation cannot be done.

The non-flat road assumption is compulsory for a robust object detection method. In literature this assumption was introduced by non-flat road approximation as series of planar surface sections $[3,4]$ or by modeling of the non-flat roads by higher order surfaces [5,6]. Many approaches perform lane detection by fitting the parameters of a 3D clothoid model using a monocular or stereo image and supplementary lane geometry constraints. The 3D lane can be used for obstacle $\mathrm{road} / \mathrm{separation}$.

This work was supported by the Romanian Ministry of Education, Research, and Youth under CNCSIS - UEFISCSU, project number PNII IDEI 1522/2008.
Disparity space-based algorithms work directly with the primary result of stereo reconstruction: the disparity map. The "V-disparity" approach [4] is widely used to detect the road surface. It has some drawbacks: is not a natural way to represent 3D (Euclidian) data, it assumes the road is dominant along the image rows, and it can be sensitive to roll angle changes (the road profile becomes blurry and harder to detect on the "V-disparity" image). One way to avoid this problem is by using a specialized vehicle with small roll variations [7]. An extended "V-disparity" approach is presented in [8], where the roll angle can be computed assuming the scene has a planar road surface and assuming the presence of high-gradient road features (edges). The "U-V-disparity" concept, introduced in [9], is used to classify the 3D road scene into relative planar surfaces and to extract the features of roadside structures and obstacles.

An approach for the detection of the road surface based on a Digital Elevation Map (DEM) representation is presented in [10]. The 3D data inferred from dense stereo are transformed into a rectangular DEM. A quadratic road surface model is initially fitted, by a RANSAC approach, to the region in front of the ego vehicle. A region growing-like process refines this primary solution, driven by the $3 \mathrm{D}$ uncertainty model of the stereo sensor. A robust global solution for the road surface is obtained. An extended and improved version of this approach was later presented in [11]. The work we will present in this paper is related to this approach, making the method more appropriate for some difficult scenarios.

\section{OVERVIEW OF THE PROPOSED IMPROVEMENTS}

Fitting a surface to a set of dense 3D points can be a time consuming task, due to the size of the data set. As already mentioned, a real-time approach for quadratic surface fitting to dense stereo data has been proposed in [11]:

1. The road surface is fitted, using a RANSAC approach, to a small patch in front of the ego vehicle. This insures the best solution is extracted for the initial set of inliers.

2. The parameters of the road surface are refined iteratively by a region growing process: new points are added to the set of inliers, if they verify the current surface and are adjacent to the current set of inliers. The parameters are re-estimated (least square 
minimization) after each iteration (when the region grows with a certain number of inliers).

This approach is computationally efficient, but it will not work robustly for some particular scenarios:

1. When the road is poorly textured and only few $3 \mathrm{D}$ points are available, the surface parameters are estimated on a small set of road inliers. It is likely that the elevation uncertainty from stereo does not have a Gaussian distribution on the reduced inliers set. Thus, the least square minimization can be biased: the most influenced parameter is the quadratic term of the surface. It will have a low accuracy, and this can corrupt the obstacle/road separation at far depths. This side effect is depicted in Fig. 1.

2. When dealing with extreme slopes (uphill or downhill roads), the road is usually composed of a quadratic surface patch at the bottom of the slope, where the vertical curvature is strong, and a planar surface patch at medium and far depths (see Fig. 2).

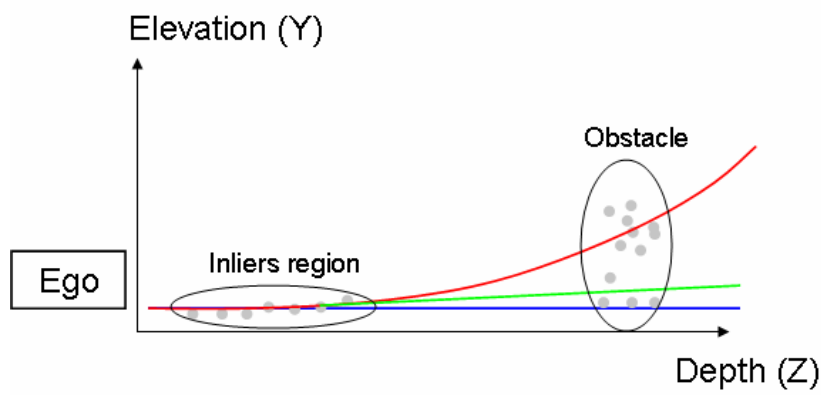

Fig. 1. A side view of a difficult scenario: the real road surface (blue), the quadratic surface (red) computed from the small set of inliers, and the planar extension of the quadratic surface (red) from the inliers region. The obstacle at far depths is incorrectly classified by the quadratic surface.

To cope with these sensitive scenarios, we propose a road model that combines a quadratic model, for the region of road inliers, with a planar surface (with green in Fig. 1) for the remaining depth range.

A key issue for scenarios with sparse 3D road reconstruction is the stabilization of the surface parameters, in order to reduce their oscillations. This can be achieved through Kalman filtering.

Two variants are investigated for the planar extension. One involves estimating the planar extension from a subset of the quadratic surface inliers, through a least-square minimization. The alternative is to estimate the planar extension based on the local slope of the quadratic surface at the location of the furthermost inliers.

\section{THE ROAD MODEL}

This proposed mixed model should perform better than the each model alone. In addition, it is more stable to sparse 3D data than a higher degree model such as cubic surfaces or Bspline curves.

The resulting surface will be composed of two main patches, a quadratic one up to the depth of the furthermost road inlier, and a planar one for the remaining 3D space of interest.
The quadratic model used is presented next, in sub-section A. In sub-section B the Kalman stabilization of the quadratic surface parameters is presented. The modeling of the planar surface used for extension is presented in sub-section $\mathrm{C}$.

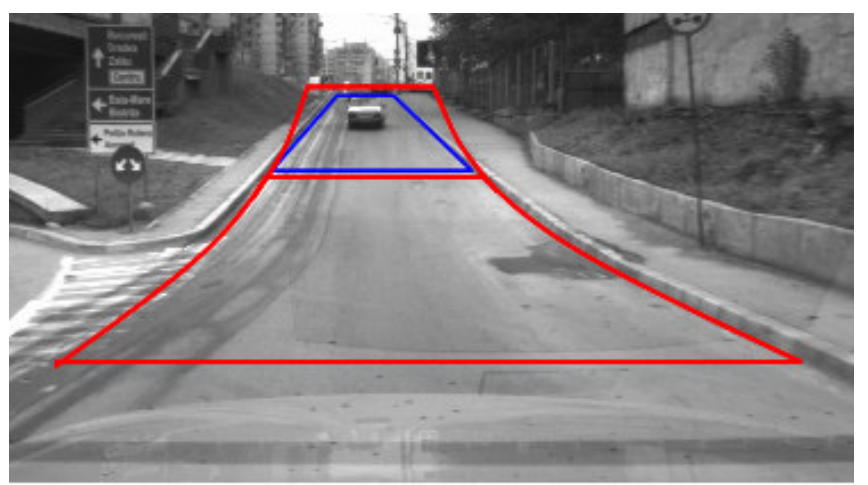

Fig. 2. When the quadratic road surface (red) is estimated at the bottom of an uphill road, its vertical curvature might cause a severe increase of the estimated road elevation at far depths. This happens if the uphill has a strong vertical curvature variation near its base, and a constant slope further away (the planar blue patch).

\section{A. The quadratic road model}

The quadratic road model allows roll and pitch angles, and quadratic variation of the elevation (the $\mathrm{Y}$ coordinate) with the depth (the longitudinal $\mathrm{Z}$ coordinate).

Equation (1) shows the algebraic form of the road model, by defining the height value $\mathrm{Y}$ with respect to the depth $\mathrm{Z}$ and the horizontal displacement $\mathrm{X}$.

$$
Y=-a \cdot X-b \cdot Z-b^{\prime} \cdot Z^{2}-c .
$$

Fitting the quadratic surface to a set of $3 \mathrm{D}$ points $(X i, Y i, Z i$, $i=1$..n) involves minimizing an error function. The error function $\mathrm{S}$ represents the sum of squared errors along the height:

$$
S=\sum_{i=1}^{n}\left(Y_{i}-Y_{R i}\right)^{2}
$$

Where $Y_{i}$ is the elevation of the 3D point $i$ and $Y_{R i}$ is the elevation of the surface at coordinates $\left(X_{i}, Z_{i}\right)$. Minimizing only along the $Y$-axis is enough because, even for curved roads, the normal of the surface is close to the Y-axis (only few degrees difference). The computational complexity is highly reduced by avoiding minimization against the normal of the surface.

By replacing (1) into (2) the following equation is obtained, where the unknowns are $a, b, b$, and $c$ :

$$
S=\sum_{i=1}^{n}\left(Y_{i}+a \cdot X_{i}+b \cdot Z_{i}+b^{\prime} \cdot Z_{i}^{2}+c\right)^{2} \text {. }
$$

The minimum of this function is 0 for perfect fitting. For $S$ to have a minimum value, its partial derivatives with respect to the unknowns must be 0 . The following system of equations must be solved: 


$$
\left\{\frac{\partial S}{\partial a}=0, \frac{\partial S}{\partial b}=0, \frac{\partial S}{\partial b^{\prime}}=0, \frac{\partial S}{\partial c}=0 .\right.
$$

After writing explicitly each equation, the system (4) becomes (matrix form):

$$
\left[\begin{array}{cccc}
S_{X^{2}} & S_{X Z} & S_{X Z^{2}} & S_{X} \\
S_{X Z} & S_{Z^{2}} & S_{Z^{3}} & S_{Z} \\
S_{X Z^{2}} & S_{Z^{3}} & S_{Z^{4}} & S_{Z^{2}} \\
S_{X} & S_{Z} & S_{Z^{2}} & n
\end{array}\right]\left[\begin{array}{c}
a \\
b \\
b^{\prime} \\
c
\end{array}\right]=\left[\begin{array}{c}
-S_{X Y} \\
-S_{Z Y} \\
-S_{Z^{2} Y} \\
-S_{Y}
\end{array}\right]
$$

Where $n$ is the number of points, and generically

$$
S_{\alpha}=\sum_{i=1}^{n} \alpha_{i}
$$

The solution of this system, if it exists, provides the quadratic road parameters.

\section{B. Quadratic Surface Stabilization}

The parameters of the quadratic surface are computed from the current stereo pair 3D data. Even if dense stereo is used to generate $3 \mathrm{D}$ data, scenarios with poor road texture (due to various reasons - scene illumination, camera quality etc.) will have fewer $3 \mathrm{D}$ road points reconstructed. The road surface will have oscillating parameters if the $3 \mathrm{D}$ set of road points is too sparse. The quadratic surface is extended (one of the variants) at far depths with a planar surface derived from the parameters of the quadratic one. Therefore, stabilization of the parameters over time must be performed.

A standard Kalman filter is applied to the parameters $[a, b$, $\left.b^{\prime}, c\right]$ of the road surface. Modeling the measurement and the process noise is the most important part that insures the stability of the Kalman filter.

Evaluating the measurement noise (matrix $R$ ) is a difficult part, because the measurements are the actual parameters of the surface. The noise in the parameters is propagated from the set of inliers used to compute the road surface. The configuration (size and area covered) of the inliers set is directly linked to the accuracy of the parameters.

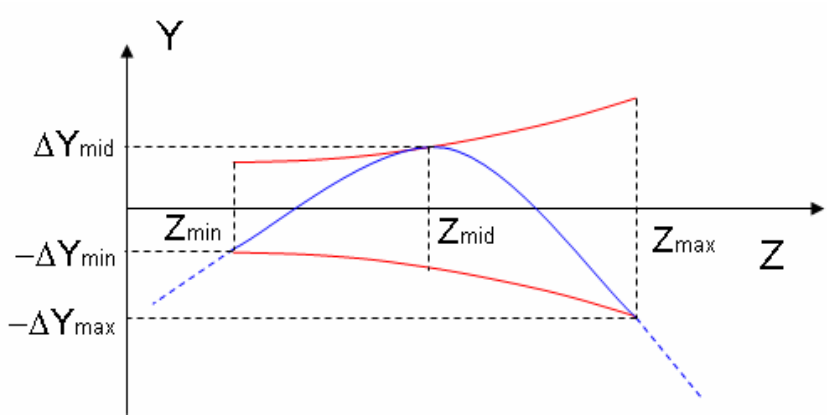

Fig. 3. The computed surface (an extreme situation, blue) should be contained within the area of uncertainty from stereo (bounded by the red curves).
Another aspect that must be considered is the correlation between the surface parameters. For instance, if a wrong curvature is estimated (on a small inliers set), then the least square fitting tends to compensate this with pitch and free term variations. This behavior can be modeled considering that the area of uncertainty along $\mathrm{Y}$, caused by the stereo reconstruction, should contain the computed surface (see Fig. 3 ). When the set of inliers is small then their uncertainty has no longer a normal distribution and extreme biases can appear in the surface shape.

The uncertainty values for the parameters $b, b^{\prime}, c$ related to the depth are computed as the parameters of the parabola that verifies the points (Zmin, $-\Delta Y$ min), (Zmid, $\Delta Y$ mid), and (Zmax, $-\Delta Y \max )$. Zmin is the $\mathrm{Z}$ coordinate of the closest inlier, $Z \max$ is the $\mathrm{Z}$ coordinate of the furthermost inlier, and $\mathrm{Zmid}$ is the middle of the inliers interval along $\mathrm{Z}$. The associated absolute values $\Delta Y$ are the uncertainties from stereo along the $\mathrm{Y}$-axis, assuming a disparity uncertainty of 0.5 pixels [11]. Thus, the parameters of this parabola provide the measurement noise:

$$
\begin{gathered}
\Delta b^{\prime}=2 \frac{\Delta Y_{\text {min }}+\Delta Y_{\text {max }}+2 \Delta Y_{\text {mid }}}{\left(Z_{\text {max }}-Z_{\text {min }}\right)^{2}} \\
\Delta b=\frac{4}{\left(Z_{\text {max }}-Z_{\text {min }}\right)^{3}}\left[\left(\Delta Y_{\text {max }}-\Delta Y_{\text {min }}\right)\left(Z_{\text {mid }}^{2}-Z_{\text {max }}^{2}\right)-\right. \\
\left.\left(\Delta Y_{\text {mid }}+\Delta Y_{\text {max }}\right)\left(Z_{\text {min }}^{2}-Z_{\text {max }}^{2}\right)\right]
\end{gathered}
$$

$$
\Delta c=-\Delta Y_{\min }-\Delta b^{\prime} Z_{\max }^{2}-\Delta b Z_{\min }
$$

The uncertainty for the roll term of the road surface is estimated in a similar way (only line fitting instead of parabola), by considering the width of the inliers set and the height uncertainty at Zmid.

The values associated with the process noise (matrix $Q$ ) are tuned in order to model the parameters variation that might occur between two successive frames:

- for $a$ : the roll angle expected variation is about 0.5 degrees,

- for $b$ : the pitch angle expected variation is about 0.5 degrees (although this extreme variation between two frames is rare, mainly due to road bumps such as speed limiters),

- for $b^{\prime}$ : the vertical curvature expected variation is chosen to insure that while approaching an uphill/downhill road (of about $10 \%$ slope), the Kalman filter allows the curvature radius to change from infinity to about 50 meters, along 1520 frames (typical approach time at a speed of 50 $\mathrm{km} / \mathrm{h}$ ), 
- for $c$ : the road elevation expected variation near the ego car is about 5 centimeters (this is also related to speed bumps).

For each frame, these values are scaled in order to compensate the variable ego speed and possible variations in the stereo acquisition frame-rate.

\section{The planar Road Model}

The planar road model used for the extension of the quadratic surface allows only roll and pitch angles. The goal of this extension is to capture the most appropriate shape of the road at far depths.

Equation (1) shows the algebraic form of the planar road model, by defining the height value $\mathrm{Y}$ with respect to the depth $\mathrm{Z}$ and the horizontal displacement $\mathrm{X}$.

$$
Y=-a \cdot X-b \cdot Z-c
$$

There are two possibilities to estimate the desired plane:

1. By least square fitting of a plane to a subset of the quadratic surface inliers.

2. Based on the local slope of the quadratic surface at location of the furthermost inliers.

The first approach involves selecting a subset $S$ of inliers with the following properties:

3. They must be the furthermost inliers from the ego car (we are interested in the local slope at far depths),

4. The subset must cover a minimal depth range (510 meters) in order to provide numerical stability to the computed plane.

Using a similar approach as in sub-section III.A, the coefficients of the plane are obtained by solving (11), applied to the subset $S$.

$$
\left[\begin{array}{ccc}
S_{X^{2}} & S_{X Z} & S_{X} \\
S_{X Z} & S_{Z^{2}} & S_{Z} \\
S_{X} & S_{Z} & n
\end{array}\right]\left[\begin{array}{l}
a \\
b \\
c
\end{array}\right]=\left[\begin{array}{l}
-S_{X Y} \\
-S_{Z Y} \\
-S_{Y}
\end{array}\right] .
$$

This variant has the main disadvantage that is does not insures elevation and slope continuity at the boundary between the two different surfaces.

The second possibility is better: the planar extension surface is build from the parameters of the quadratic surface. It has the same roll angle, and the pitch angle is equal to the pitch angle of quadratic surface at its furthermost inlier. Assuming the furthermost inlier is placed at the depth $Z \max$, the local slope of the quadratic surface is obtained by deriving (1) with respect to $Z$ :

$$
b_{\text {planar }}=\frac{d Y}{d Z}=-b_{\text {quadratic }}-2 b_{\text {quadratic }}^{\prime} \cdot Z_{\max } .
$$

The free term of the plane is computed by considering that at the depth Zmax, the quadratic and the planar surface must have the same elevation.

\section{The Mixed Road Model}

To summarize the discussion, the mixed model proposed uses a quadratic surface $\left(a q, b q, b^{\prime} q, c q\right)$ up to the depth of the furthermost road inlier, and a planar surface $(a p, b p, c p)$ further away (Fig. 4). The road elevation at a given 3D location $(X, Z)$ is given by:

$$
Y=\left\{\begin{array}{cc}
-a_{q} \cdot X-b_{q} \cdot Z-b_{q}^{\prime} \cdot Z^{2}-c_{q}, & \text { if } Z<Z_{\max } \\
-a_{p} \cdot X-b_{p} \cdot Z-c_{p}, \quad \text { otherwise }
\end{array} .\right.
$$

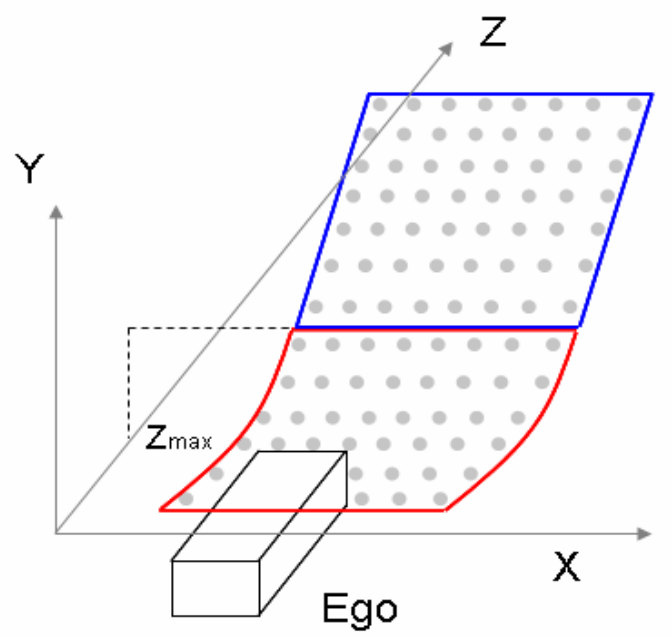

Fig. 4. The mixed road model proposed.

\section{IMPLEMENTATION DETAILS}

Building the DEM and the computation of the quadratic surface parameters on the DEM follows the steps described in [11]:

- For each DEM cell, the elevation of the highest point (within the cell) is stored, or the cell is marked empty. A DEM cell covers a 10x10 cm patch in the horizontal plane. Singular empty cells are filled with the neighbors' elevation, in order to improve the connectivity in the DEM.

- The quadratic road surface is fitted, using a RANSAC approach, to a small DEM patch in front of the ego vehicle.

- The parameters of the road surface are refined iteratively by a region growing process: new points are added tot the set of inliers and the parameters are re-estimated after each iteration. 
The Kalman filter is applied to the quadratic road surface for stabilization (described in section III.B). The planar extension is estimated from the parameters of the quadratic surface (described in section III.C).

Finally, the parameters of the mixed model are transformed from the DEM reference frame into the world reference frame and the road/obstacle separation is performed. The elevation of the road for each $3 \mathrm{D}$ point $(X, Z)$ location is compared with the elevation of the $3 \mathrm{D}$ point. $3 \mathrm{D}$ points that are above the road surface with a certain elevation difference are considered candidate obstacle points for higher-level modules (see Fig. 5). The minimum elevation difference is provided by the uncertainty of the stereo sensor [11] and can be computed as a function of the focal length, stereo baseline, depth, and disparity uncertainty.

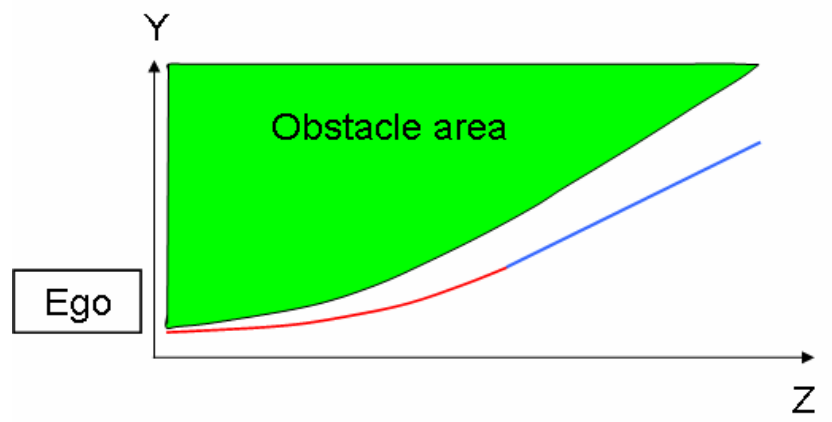

Fig. 5. Obstacle area defined by the mixed road model.
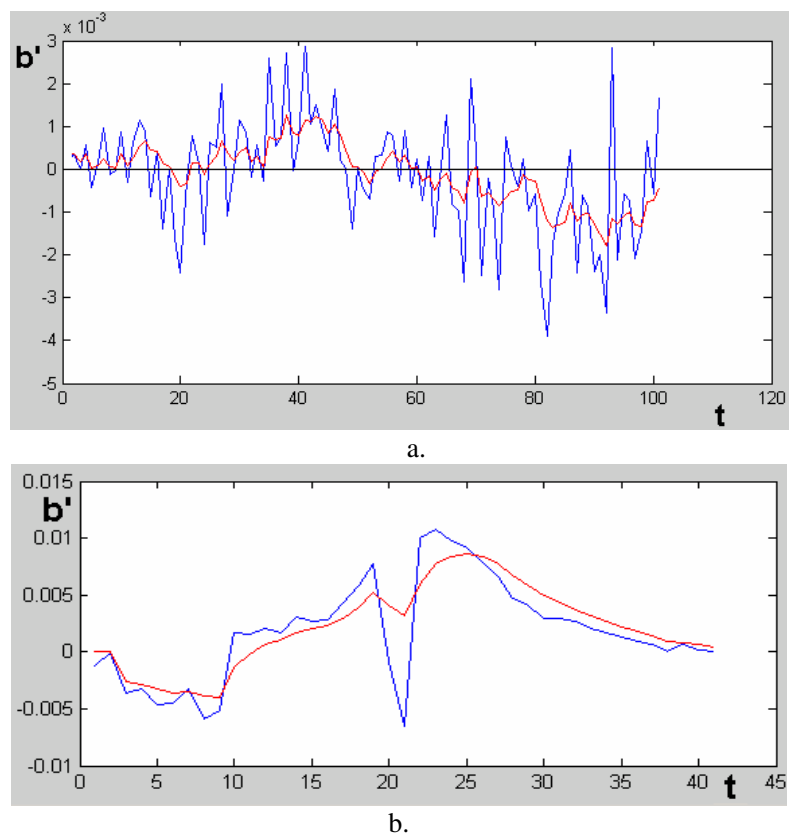

Fig. 6. a. The measured (blue) versus the filtered (red) curvature term b' are shown for 120 frames while driving on a relatively flat road, with poor 3D reconstruction, $\mathrm{b}$. The measured (blue) versus the filtered (red) curvature term b' are shown for 45 frames while driving toward a $10 \%$ uphill road.

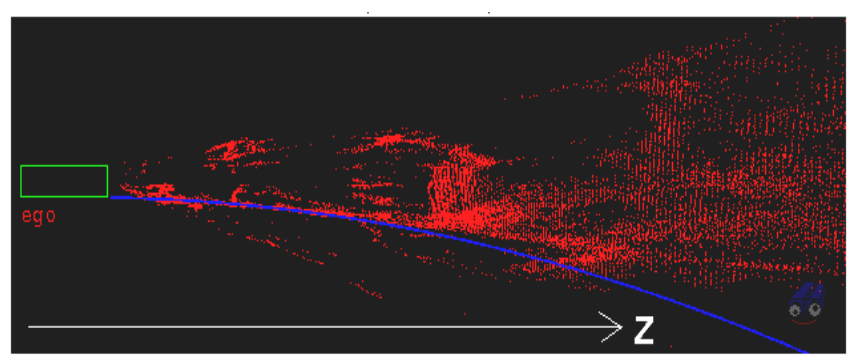

a.

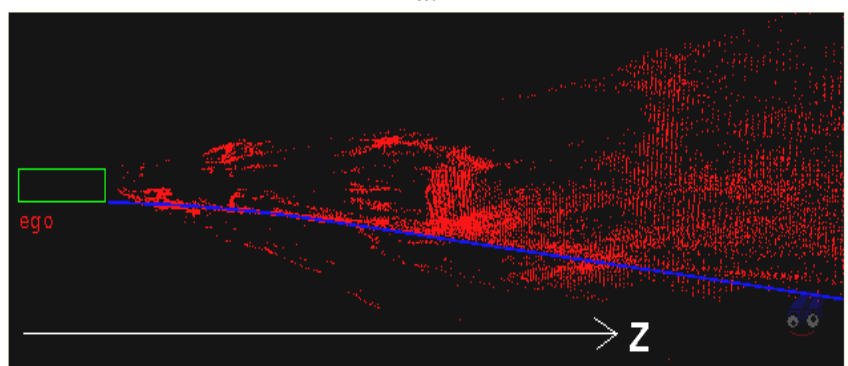

b.

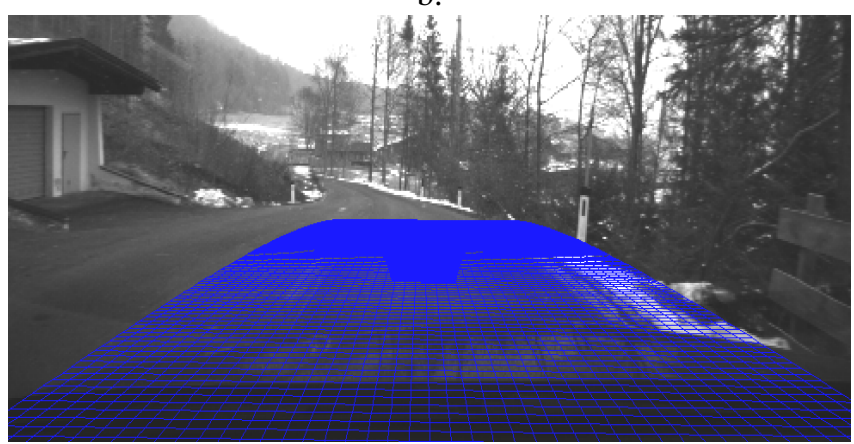

c.

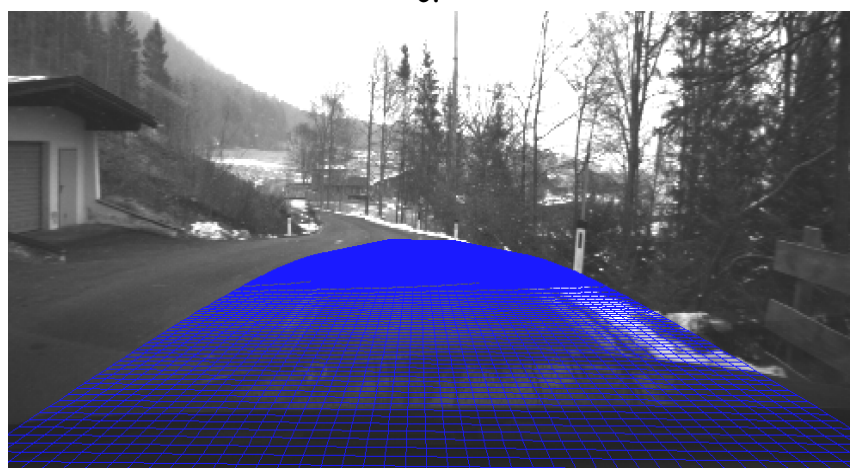

d.

Fig. 7. a. Side view of the quadratic road model and the set of 3D points, $b$. Side view of the mixed road model and the set of 3D points, $c$. The quadratic road surface projected onto the left image, $d$. The mixed model surface projected onto the left image.

\section{RESUlTS AND FUTURE WORK}

The algorithm was tested both offline and online with an onboard dense stereo system. It runs in real-time, in about than 7-10 ms per frame. Implementation was done in $\mathrm{C}++$, and the tests were carried out on a Pentium Dual-Core processor.

The evaluation of the Kalman filtering was performed in two directions: to test the stability of the filter on scenarios with reduced number of road points and to evaluate its ability 
to converge to the true road shape with minimal delay.

First, the filtering was evaluated on a relatively flat road, with poor texture. The graph showing the measured curvature term versus the filtered one (see Fig. 6.a) proves that the filter is able to remove most of the measurement noise.

The second graph (see Fig. 6.b) shows the filtered curvature while driving towards an uphill road. The road has a good 3D reconstruction, and the filter is able to quickly converge. However, in frames 20-22 the windshield wipers were on, partially obscuring the stereo system. A poor set of road inliers was available, but the filter successfully filtered the wrong measurements due to the adaptive way of estimating the measurement noise (as a function of the inliers' set).

As expected, the mixed road model performs better than the quadratic model alone in most of the scenarios (an example in Fig. 7) in terms of separating points into road or obstacle points. Various results of the obstacle/road separation are shown in Fig. 8. The improvements are visible at far depths, where the mixed road model is less likely to perform wrong classifications.

\section{REFERENCES}

[1] J.Weber, D. Koller, Q.-T. Luong and J. Malik, "An integrated stereo-based approach to automatic vehicle guidance," Fifth International Conference on Computer Vision, 20-23 June 1995, Cambridge, MA, USA, pp.52-57.

[2] T. A. Williamson, "A high-performance stereo vision system for obstacle detection," PhD Thesis CMU-RI-TR-98-24, Robotics Institute Carnegie Mellon University, Pittsburg, September, 1998.

[3] J. Hancock, "High-Speed Obstacle Detection for Automated Highway Applications," Tech. Report CMU-RI-TR-97-17, Robotics Institute, Carnegie Mellon University, Pittsburg, May, 1997.

[4] R. Labayrade, D. Aubert, J.P. Tarel, "Real Time Obstacle Detection in Stereovision on Non Flat Road Geometry Through V-disparity Representation," Proceedings of IEEE Intelligent Vehicle Symposium (IV'2002), June 18-20, 2002, Versailles, France.

[5] R. Aufrere, R. Chapuis, F. Chausse, "A model-driven approach for real-time road recognition," Machine Vision and Applications, Springer-Verlag, 2001, pp. 95-107.

[6] J. Goldbeck, B. Huertgen, "Lane Detection and Tracking by Video Sensors," In Proc.of IEEE International Conference on Intelligent Transportation Systems, October 5-8, 1999, Tokyo Japan, pp. 74-79.

[7] A. Broggi, C. Caraffi, P. Paolo Porta, and P. Zani, "The Single Frame Stereo Vision System for Reliable Obstacle Detection used during the 2005 DARPA Grand Challenge on TerraMax," in IEEE Intelligent Transportation Systems Conference, Toronto, Canada, pp. 745-752, September 17-20, 2006.

[8] R. Labayrade and D. Aubert, "A single framework for vehicle roll, pitch, yaw estimation and obstacles detection by stereovision," IEEE Intelligent Vehicles Symposium, Columbus, USA, pp. 31 - 36, June 2003.

[9] Z. Hu and K. Uchimura, "U-V-Disparity: An efficient algorithm for stereovision based scene analysis," in IEEE Intelligent Vehicles Symposium, Las Vegas, USA, pp. 48-54, June 2005.

[10] F. Oniga, S. Nedevschi, M-M. Meinecke, T-B. To, "Road Surface and Obstacle Detection Based on Elevation Maps from Dense Stereo," the 10th International IEEE Conference on Intelligent Transportation Systems, Sept. 30 - Oct. 3, 2007, Seattle, Washington, USA.
[11] F. Oniga, S. Nedevschi, "Processing Dense Stereo Data Using Elevation Maps: Road Surface, Traffic Isle, and Obstacle Detection," IEEE Transactions on Vehicular Technology, March 2010.
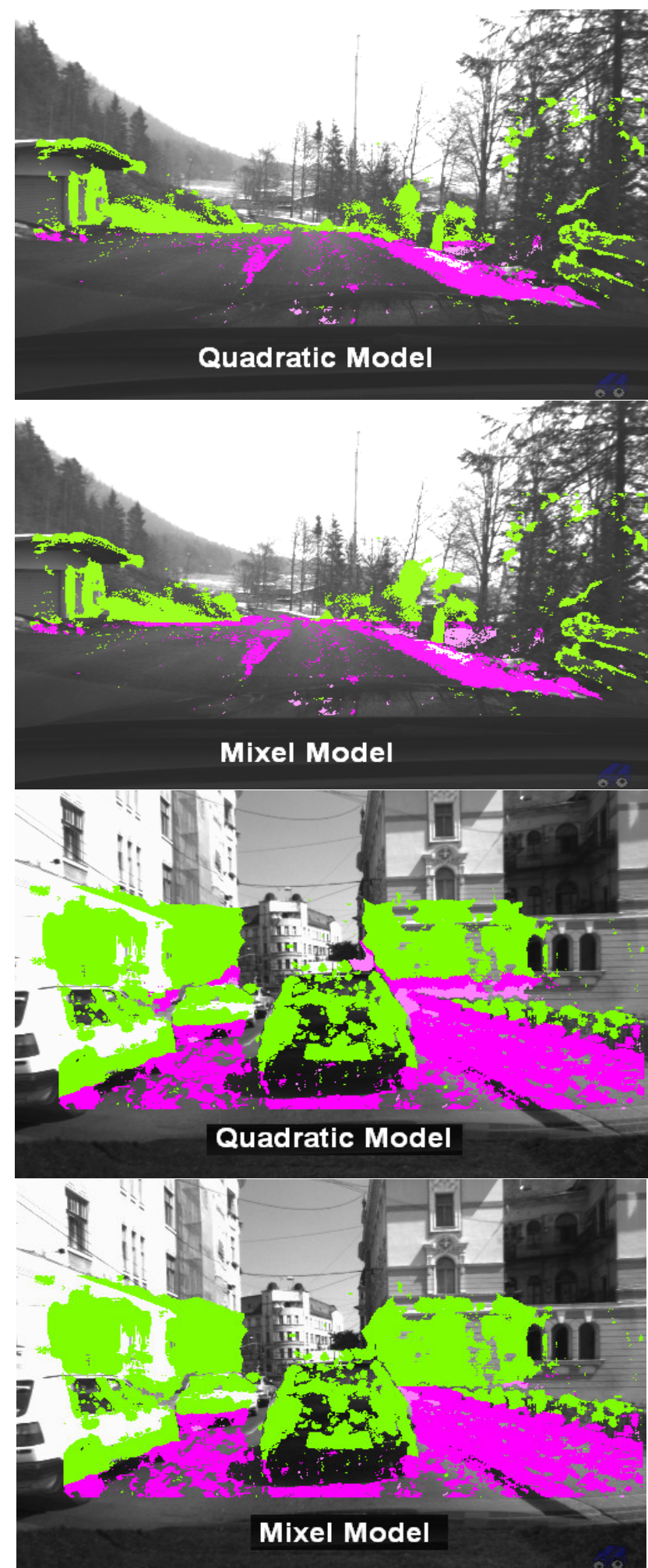

Fig. 8. Obstacle road separation works better at far depths with the mixed road model: obstacle points with green and road points with magenta. 